doi: $10.2306 /$ scienceasia1513-1874.2013.39.312

\title{
A relaxation approximation of the incompressible Navier-Stokes system
}

\author{
Hongli Wang ${ }^{\mathrm{a}, *}$, Huimin Liu ${ }^{\mathrm{b}}$ \\ a College of Mathematics and Information Science, \\ North China University of Water Resources and Electric Power, Zhengzhou 450011, China \\ b General Subjects Department, Zhengzhou Chenggong University of Finance and Economics, \\ Zhengzhou 451200, China
}

*Corresponding author, e-mail: wanghongli@ncwu.edu.cn

Received 17 May 2012

Accepted 20 Mar 2013

ABSTRACT: In this article, we consider a hyperbolic singular perturbation of the incompressible Navier-Stokes equations in a $d$-dimensional unit periodic square. For well-prepared periodic initial data, we give a rigorous justification of the diffusion relaxation limit towards the Navier-Stokes equations by using the energy method.

KEYWORDS: incompressible Navier-Stokes equations, relaxation approximations, singular perturbations, energy method

\section{INTRODUCTION}

Let us consider the following system ${ }^{1}$ :

$$
\begin{aligned}
\partial_{t} \widetilde{u}+\operatorname{div} \widetilde{V} & =\nabla \widetilde{\phi}, \\
\partial_{t} \widetilde{V}+\nabla \widetilde{u} & =-\frac{1}{\epsilon}(\widetilde{V}-\widetilde{u} \otimes \widetilde{u}), \\
\operatorname{div} \widetilde{u} & =0,
\end{aligned}
$$

for $(x, t) \in \Omega \times[0, T]$, where $\Omega=(0,1]^{d}$ is the unit periodic square. The unknowns are $\widetilde{u} \in \mathbb{R}^{d}, \widetilde{V} \in \mathbb{R}^{d, d}$ and $\widetilde{\phi} \in \mathbb{R}$. Let us notice that, as $\epsilon \rightarrow 0$, we formally obtain the incompressible system

$$
\left\{\begin{array}{l}
\partial_{t} \widetilde{u}+\operatorname{div}(\widetilde{u} \otimes \widetilde{u})=\nabla \widetilde{\phi} \\
\operatorname{div} \widetilde{u}=0 .
\end{array}\right.
$$

Now, let us consider a diffusive relaxation scaling, namely, for $\epsilon>0$, we set

$$
\begin{array}{r}
\widetilde{u}(x, t)=\epsilon u(x, \epsilon t), \\
\widetilde{V}(x, t)=\epsilon V(x, \epsilon t), \\
\widetilde{\phi}(x, t)=\epsilon^{2} \phi(x, \epsilon t) .
\end{array}
$$

Hence system (1) becomes

$$
\begin{gathered}
\partial_{t} u+\frac{1}{\epsilon} \operatorname{div} V=\nabla \phi, \\
\partial_{t} V+\frac{1}{\epsilon} \nabla u=-\frac{V}{\epsilon^{2}}+\frac{u \otimes u}{\epsilon}, \\
\operatorname{div} u=0 .
\end{gathered}
$$

Applying the Maxwell iteration to (2b) gives

$$
\begin{aligned}
V & =-\epsilon \nabla u+\epsilon(u \otimes u)-\epsilon^{2} \partial_{t} V \\
& =-\epsilon \nabla u+\epsilon(u \otimes u)+O\left(\epsilon^{3}\right) .
\end{aligned}
$$

Substituting the truncation $V=-\epsilon \nabla u+\epsilon(u \otimes u)$ into (2a), we arrive at the incompressible Navier-Stokes equations

$$
\begin{aligned}
\partial_{t} u+\operatorname{div}(u \otimes u)-\Delta u & =\nabla \phi, \\
\operatorname{div} u & =0 .
\end{aligned}
$$

The goal of this paper is to justify the above formal derivation of the incompressible Navier-Stokes equations for periodic IVPs (initial-value problems) with an emphasis on several space dimensions.

By using the modulated energy method, the diffusive and relaxation limit of system (1) has been investigated $^{1}$, whose techniques were restricted to the 2 -d case. With the help of the hyperbolic energy methods for studying incompressible fluids, the above limit for the compressible version of system (1) is studied $^{2}$. These papers all give a rigorous justification of its asymptotic limit towards the incompressible Navier-Stokes equations. For other diffusive relaxation models and approximations, we refer to Refs. 3-7 and the references therein for this topic.

In this paper, we study the relaxation limit problem of system (2) in the different scaling from ${ }^{1}$ and obtain the different convergence result. Precisely, we assume that incompressible Navier-Stokes (3) have a smooth solution $(u, \phi)$ with initial data $u(x, 0)=$ 
$u_{0}(x)$. Inspired by the Maxwell iteration above, we construct a formal approximation

$$
\left(u_{\epsilon}, V_{\epsilon}, \phi_{\epsilon}\right)=(u,-\epsilon \nabla u+\epsilon(u \otimes u), \phi)
$$

for the solution $\left(u^{\epsilon}, V^{\epsilon}, \phi^{\epsilon}\right)$ of (2) with initial data

$$
(u(x, 0), V(x, 0))=\left(u_{0}(x),-\epsilon \nabla u_{0}+\epsilon\left(u_{0} \otimes u_{0}\right)\right) .
$$

Then, we will use energy methods to prove that $\left(u^{\epsilon}, V^{\epsilon}, \phi^{\epsilon}\right)$ exists in the finite time interval where $u$ is well defined and $\left(u^{\epsilon}, V^{\epsilon}, \phi^{\epsilon}\right)$ can be expressed as

$$
\left(u^{\epsilon}, V^{\epsilon}, \phi^{\epsilon}\right)=\left(u_{\epsilon}, V_{\epsilon}, \phi_{\epsilon}\right)+O\left(\epsilon^{2}\right)
$$

in the Sobolev space $H^{s}(\Omega)$ with $s>\frac{d}{2}+1$.

Now we recall some results on the Moser-type calculus inequalities in Sobolev spaces, and the local existence of smooth solutions for symmetrizable hyperbolic equations for later use in this paper.

Lemma 1 (Moser-type calculus inequalities, Refs. 8, 9). Let $s \geqslant 1$ be an integer. Suppose $u \in H^{s}\left(\mathcal{T}^{3}\right)$, $\nabla u \in L^{\infty}\left(\mathcal{T}^{3}\right)$, and $v \in H^{s-1}\left(\mathcal{T}^{3}\right) \cap L^{\infty}\left(\mathcal{T}^{3}\right)$. Then for all multi-indexes $|\alpha| \leqslant s$, we have $\left(\partial_{x}^{\alpha}(u v)-\right.$ $\left.u \partial_{x}^{\alpha} v\right) \in L^{2}\left(\mathcal{T}^{3}\right)$ and

$$
\begin{aligned}
& \left\|\partial_{x}^{\alpha}(u v)-u \partial_{x}^{\alpha} v\right\| \\
& \leqslant C_{s}\left(\|\nabla u\|_{0, \infty}\left\|D^{|\alpha|-1} v\right\|+\left\|D^{|\alpha|} u\right\|\|v\|_{0, \infty}\right),
\end{aligned}
$$

where

$$
\left\|D^{h} u\right\|=\sum_{|\alpha|=h}\left\|\partial_{x}^{\alpha} u\right\|, \forall h \in \mathbb{N} .
$$

Moreover, if $s \geqslant 3$, then the embedding $H^{s-1}\left(\mathcal{T}^{3}\right) \hookrightarrow$ $L^{\infty}\left(\mathcal{T}^{3}\right)$ is continuous and we have

$$
\begin{aligned}
& \|u v\|_{s-1} \leqslant C_{s}\|u\|_{s-1}\|v\|_{s-1}, \\
& \left\|\partial_{x}^{\alpha}(u v)-u \partial_{x}^{\alpha} v\right\| \leqslant C_{s}\|u\|_{s}\|v\|_{s-1} .
\end{aligned}
$$

\section{PRELIMINARIES AND FORMAL APPROXIMATIONS}

First we shall state the existence of smooth local solutions for system (2) (See Ref. 1).

Theorem 1 Let $s>\frac{d}{2}+1$ be an integer. Suppose the initial data $\left(u_{0}(x), V_{0}(x)\right)$ are smooth functions belonging to $H^{s}(\Omega)$. Then, there exists a positive time $T^{\epsilon}$, which depends only on the initial data, and a solution $\left(u^{\epsilon}, V^{\epsilon}, \phi^{\epsilon}\right) \in C\left(\left[0, T^{\epsilon}\right] ;\left(H^{s}(\Omega)\right)^{3}\right)$ to system (2). Moreover, if $T^{\epsilon}<\infty$, then

$$
\lim _{t \rightarrow T^{\epsilon}}\left\|\left(u^{\epsilon}, V^{\epsilon}\right)\right\|_{s}=\infty .
$$

The proof follows easily by arguing as for the classical wave equation, by using energy estimates and the Gagliardo-Nirenberg inequalities, see for instance Ref. 10, and it is omitted.

Let $(u, \phi)$ solve the IVP of the incompressible Navier-Stokes (3). Inspired by the Maxwell iteration, we take

$$
\left(u_{\epsilon}, V_{\epsilon}, \phi_{\epsilon}\right)=(u,-\epsilon \nabla u+\epsilon(u \otimes u), \phi) .
$$

Define

$$
R_{\epsilon}=\frac{\partial_{t} V_{\epsilon}}{\epsilon}=-\partial_{t}(\nabla u-u \otimes u) .
$$

Then we have

$$
\begin{aligned}
\partial_{t} u_{\epsilon}+\frac{1}{\epsilon} \operatorname{div} V_{\epsilon} & =\nabla \phi_{\epsilon}, \\
\partial_{t} V_{\epsilon}+\frac{1}{\epsilon} \nabla u_{\epsilon} & =-\frac{V_{\epsilon}}{\epsilon^{2}}+\frac{u_{\epsilon} \otimes u_{\epsilon}}{\epsilon}+\epsilon R_{\epsilon}, \\
\operatorname{div} u_{\epsilon} & =0 .
\end{aligned}
$$

Obviously, (4) is equivalent incompressible NavierStokes (3).

Recalling the classical result ${ }^{11,12}$ on the existence of sufficiently regular solutions of the incompressible Navier-Stokes (3), we have the following regularity result about $\left(u_{\epsilon}, V_{\epsilon}, \phi_{\epsilon}\right)$.

Lemma 2 Let $u_{0}$ satisfy $u_{0} \in H^{s+3}$ and $\operatorname{div} u_{0}=0$ for $s>d / 2+1$. Then there exist $0<T_{*} \leqslant \infty$ (ifd $=$ $\left.2, T_{*}=\infty\right)$, the maximal existence time, such that

$$
\begin{array}{r}
\sup _{t \in\left[0, T_{0}\right]}\left(\left\|u_{\epsilon}\right\|_{s+2}+\left\|\nabla \phi_{\epsilon}\right\|_{s+2}+\left\|V_{\epsilon}\right\|_{s+1}+\left\|R_{\epsilon}\right\|_{s}\right) \\
\leqslant C\left(T_{0}\right)
\end{array}
$$

for any $T_{0}<T_{*}$.

\section{THE MAIN RESULT}

Having constructed the formal approximation $\left(u_{\epsilon}, V_{\epsilon}, \phi_{\epsilon}\right)$ for the periodic IVP of the system (2), we prove here the validity of the approximation under some regularity assumptions on the given data and an existence result for the IVP. The main result of this paper is stated as follows.

Theorem 2 Let $s \in \mathbb{N}$ with $s \geqslant \frac{d}{2}+1$. Suppose that the incompressible Navier-Stokes (3) have a solution $\left(u_{\epsilon}, \phi_{\epsilon}\right)$ satisfying (5).

Then, for $\epsilon$ sufficiently small, problem (2) with periodic initial data

$$
\begin{aligned}
& (u(x, 0), V(x, 0)) \\
& \quad=\left(u_{0}(x),-\epsilon \nabla u_{0}(x)+\epsilon\left(u_{0}(x) \otimes u_{0}(x)\right)\right)
\end{aligned}
$$


has a unique solution $\left(u^{\epsilon}, V^{\epsilon}\right) \in C\left(\left[0, T_{*}\right], H^{s}(\Omega)\right)$, and there exists a constant $C_{1}>0$, independent of $\epsilon$ but dependent on $T_{*}$, such that

$$
\left\|\left(u^{\epsilon}-u_{\epsilon}, V^{\epsilon}-V_{\epsilon}\right)(\cdot, t)\right\|_{s} \leqslant C_{1} \epsilon^{2}, \forall t \in\left[0, T_{*}\right] .
$$

Moreover,

$$
\left\|V^{\epsilon}-V_{\epsilon}\right\|_{L^{2}\left(\left[0, T_{*}\right], H^{s}(\Omega)\right)} \leqslant C_{1} \epsilon^{3} .
$$

Proof: First, let us set the error

$$
\left(E^{u}, E^{V}, E^{\phi}\right)=\left(u^{\epsilon}-u_{\epsilon}, V^{\epsilon}-V_{\epsilon}, \phi^{\epsilon}-\phi_{\epsilon}\right) .
$$

From the equations in (2) and (4), it follows that the error $\left(E^{u}, E^{V}, E^{\phi}\right)$ satisfies

$$
\left\{\begin{array}{l}
\partial_{t} E^{u}+\frac{1}{\epsilon} \operatorname{div} E^{V}=\nabla E^{\phi}, \\
\partial_{t} E^{V}+\frac{1}{\epsilon} \nabla E^{u}=-\frac{E^{V}}{\epsilon^{2}} \\
\quad+\frac{1}{\epsilon}\left(\left(E^{u}+u_{\epsilon}\right) \otimes E^{u}+E^{u} \otimes u_{\epsilon}\right)-\epsilon R_{\epsilon}, \\
\operatorname{div} E^{u}=0 .
\end{array}\right.
$$

We differentiate the above equation with $\partial_{x}^{\alpha}$ for a multi-index $\alpha$ satisfying $|\alpha| \leqslant s$ with $s>\frac{d}{2}+1$ to get

$$
\begin{aligned}
\partial_{t} E_{\alpha}^{u}+\frac{1}{\epsilon} \operatorname{div} E_{\alpha}^{V} & =\nabla E_{\alpha}^{\phi} \\
\partial_{t} E_{\alpha}^{V}+\frac{1}{\epsilon} \nabla E_{\alpha}^{u} & =-\frac{E_{\alpha}^{V}}{\epsilon^{2}}+\frac{1}{\epsilon} F_{\alpha}-\epsilon R_{\epsilon \alpha}, \\
\operatorname{div} E_{\alpha}^{u} & =0
\end{aligned}
$$

where

$$
F_{\alpha}=\left[\left(E^{u}+u_{\epsilon}\right) \otimes E^{u}+E^{u} \otimes u_{\epsilon}\right]_{\alpha} .
$$

Before performing the energy estimate, we set

$$
\begin{aligned}
\mathcal{E}_{\alpha, s}(t) & =\left\|E_{\alpha}^{u}(t)\right\|^{2}+\left\|E_{\alpha}^{V}(t)\right\|^{2}, \\
\mathcal{E}_{s}(t) & =\sum_{|\alpha| \leqslant s} \mathcal{E}_{\alpha, s}(t) .
\end{aligned}
$$

Taking the $L^{2}$ inner product of the first equation in (6) with $E_{\alpha}^{u}$, one gets, by using the third equation in (6) and integration by parts that

$$
\begin{aligned}
\frac{1}{2} \frac{\mathrm{d}}{\mathrm{d} t}\left\|E_{\alpha}^{u}\right\|^{2} & =-\frac{1}{\epsilon} \int_{\Omega} E_{\alpha}^{u} \operatorname{div} E_{\alpha}^{V} \mathrm{~d} x+\int_{\Omega} E_{\alpha}^{u} \nabla E_{\alpha}^{\phi} \mathrm{d} x \\
& =-\frac{1}{\epsilon} \int_{\Omega} E_{\alpha}^{u} \operatorname{div} E_{\alpha}^{V} \mathrm{~d} x .
\end{aligned}
$$

Taking the $L^{2}$ inner product of the second equation in (6) with $E_{\alpha}^{V}$, one gets, by integration by parts and
Cauchy-Schwarz's inequality that

$$
\begin{aligned}
\frac{1}{2} \frac{\mathrm{d}}{\mathrm{d} t} \| & E_{\alpha}^{V} \|^{2}+\frac{1}{\epsilon^{2}} \int_{\Omega}\left|E_{\alpha}^{V}\right|^{2} \mathrm{~d} x \\
= & -\frac{1}{\epsilon} \int_{\Omega} E_{\alpha}^{V} \nabla E_{\alpha}^{u} \mathrm{~d} x+\frac{1}{\epsilon} \int_{\Omega} E_{\alpha}^{V} F_{\alpha} \mathrm{d} x \\
& \quad-\epsilon \int_{\epsilon \alpha} E_{\alpha}^{V} \mathrm{~d} x \\
= & \frac{1}{\epsilon} \int_{\Omega} E_{\alpha}^{u} \operatorname{div} E_{\alpha}^{V} \mathrm{~d} x+\frac{1}{\epsilon} \int_{\Omega} E_{\alpha}^{V} F_{\alpha} \mathrm{d} x \\
& \quad-\epsilon \int_{\Omega} E_{\alpha}^{V} R_{\epsilon \alpha} \mathrm{d} x \\
\leqslant & \frac{1}{\epsilon} \int_{\Omega} E_{\alpha}^{u} \operatorname{div} E_{\alpha}^{V} \mathrm{~d} x+\frac{1}{2 \epsilon^{2}} \int_{\Omega}\left|E_{\alpha}^{V}\right|^{2} \mathrm{~d} x \\
& +C \int_{\Omega}\left|F_{\alpha}\right|^{2} \mathrm{~d} x+C \epsilon^{4},
\end{aligned}
$$

where, we have used the boundedness of the $\left\|R_{\epsilon}\right\|_{s}$ in inequality (5). Then, we have

$$
\begin{aligned}
& \frac{1}{2} \frac{\mathrm{d}}{\mathrm{d} t}\left\|E_{\alpha}^{V}\right\|^{2}+\frac{1}{2 \epsilon^{2}}\left\|E_{\alpha}^{V}\right\|^{2} \\
& \quad \leqslant \frac{1}{\epsilon} \int_{\Omega} E_{\alpha}^{u} \operatorname{div} E_{\alpha}^{V} \mathrm{~d} x+C\left\|F_{\alpha}\right\|^{2}+C \epsilon^{4} .
\end{aligned}
$$

From (7) and (8), we have

$$
\frac{\mathrm{d}}{\mathrm{d} t} \mathcal{E}_{\alpha, s}(t)+\frac{1}{\epsilon^{2}}\left\|E_{\alpha}^{V}\right\|^{2} \leqslant C\left\|F_{\alpha}\right\|^{2}+C \epsilon^{4} .
$$

For $F_{\alpha}$, the Moser-type calculus inequality gives

$$
\begin{aligned}
\left\|F_{\alpha}\right\|^{2} & \leqslant C\left(\left(1+\left\|E^{u}\right\|_{s}\right)\left\|E^{u}\right\|_{s}\right)^{2} \\
& \leqslant C\left(1+\mathcal{E}_{s}(t)\right) \mathcal{E}_{s}(t) .
\end{aligned}
$$

Then, Young inequality gives

$$
\frac{\mathrm{d}}{\mathrm{d} t} \mathcal{E}_{\alpha, s}(t)+\frac{1}{\epsilon^{2}}\left\|E_{\alpha}^{V}\right\|^{2} \leqslant C\left(1+\mathcal{E}_{s}(t)\right) \mathcal{E}_{s}(t)+C \epsilon^{4} .
$$

Integrating (9) over $(0, t)$ with $t \in(0, T], T=$ $\min \left\{T^{\epsilon}, T *\right\}$ and summing up over $\alpha$ satisfying $|\alpha| \leqslant$ $s$, we obtain

$$
\begin{aligned}
\mathcal{E}_{s}(t)+ & \frac{1}{\epsilon^{2}} \int_{0}^{t}\left\|E^{V}(\xi)\right\|_{s}^{2} \mathrm{~d} \xi \\
& =C \int_{0}^{t}\left(1+\mathcal{E}_{s}(\xi)\right) \mathcal{E}_{s}(\xi) \mathrm{d} \xi+C \epsilon^{4} .
\end{aligned}
$$

Now we let

$$
z(t)=C \int_{0}^{t}\left(1+\mathcal{E}_{s}(\xi)\right) \mathcal{E}_{s}(\xi) \mathrm{d} \xi+C \epsilon^{4} .
$$


Then it follows from (10) that

$\mathcal{E}_{s}(t) \leqslant z(t), \frac{1}{\epsilon^{2}} \int_{0}^{t}\left\|E^{V}(\xi)\right\|_{s}^{2} \mathrm{~d} \xi \leqslant z(t), \forall t \in(0, T]$,

and

$$
z^{\prime}(t)=C\left(1+\mathcal{E}_{s}(t)\right) \mathcal{E}_{s}(t) \leqslant C z(t)(1+z(t)),
$$

with

$$
z(0)=C \epsilon^{4} .
$$

A straightforward computation yields

$$
z(t) \leqslant C \epsilon^{4} \mathrm{e}^{C t} \leqslant C \epsilon^{4} \mathrm{e}^{C T_{*}}, \forall t \in(0, T] .
$$

Hence from (11) we obtain

$$
\begin{gathered}
\mathcal{E}_{s}(t) \leqslant z(t) \leqslant C \epsilon^{4}, \\
\int_{0}^{t}\left\|E^{V}(\xi)\right\|_{s}^{2} \mathrm{~d} \xi \leqslant \epsilon^{2} z(t) \leqslant C \epsilon^{6},
\end{gathered}
$$

$\forall t \in(0, T]$. In particular, this implies that $\left(E^{u}, E^{V}\right)$ is bounded in $L^{\infty}\left([0, T], H^{s}(\Omega)\right)$. By a standard argument on the time extension of smooth solutions, we obtain $T^{\epsilon} \geqslant T_{*}$, i.e., $T=T_{*}$. This completes the proof of Theorem 2 .

Acknowledgements: This work was supported by the United Fund for Fostering Talents of NSFC-Henan (Grant No. U1204103) and the science and technology research projects of Education Department of Henan province (Grant No. 13A110731).

\section{REFERENCES}

1. Brenier Y, Natalini R, Puel M (2004) On a relaxation approximation of the incompressible Navier-Stokes equations. Proc Am Math Soc 132, 1021-8.

2. Natalini R, Rousset F (2006) Convergence of a singular Euler-Poisson approximation of the incompressible Navier-Stokes equations. Proc Am Math Soc 134, 2251-8.

3. Yong W (2004) Diffusive relaxation limit of multidimensional isentropic hydrodynamic models for semiconductors. SIAM J Appl Math 64, 1737-48.

4. Jüngel A, Peng Y (1999) A hierarchy of hydrodynamic models for plasmas zero-relaxation-time limits. Comm Part Differ Equat 24, 1007-33.

5. Lattanzio C, Marcati P (1999) The relaxation to the drift-diffusion system for the 3-D isentropic EulerPoisson model for semiconductors. Discrete Contin Dyn Syst 5, 449-55.

6. Xu J, Yong W (2009) Relaxation-time limits of nonisentropic hydrodynamic models for semiconductors. J Differ Equat 247, 1777-95.

7. Yang J, Wang S, Zhao J (2011) The relaxation-time limit in the compressible Euler-Maxwell equations. Nonlin Anal 74, 7005-11.
8. Klainerman S, Majda A (1981) Singular limits of quasilinear hyperbolic systems with large parameters and the incompressible limit of compressible fluids. Comm Pure Appl Math 34, 481-524.

9. Majda A (1984) Compressible Fluid Flow and Systems of Conservation Laws in Several Space Variables. Springer-Verlag, New York.

10. Klainerman S (1980) Global existence for nonlinear wave equations. Comm Pure Appl Math 33, 43-101.

11. McGrath F (1968) Nonstationary plane flow of viscous and ideal fluids. Arch Ration Mech Anal 27, 229-348.

12. Kato T (1972) Nonstationary flow of viscous and ideal fluids in $R^{3}$. J Funct Anal 9, 296-305. 